\title{
Encefalopatia espongiforme bovina atípica: uma revisão
}

\section{Atypical bovine spongiform encephalopathy: a review}

\author{
Ellen Elizabeth Laurindo ${ }^{*}$, Ivan Roque de Barros Filho²

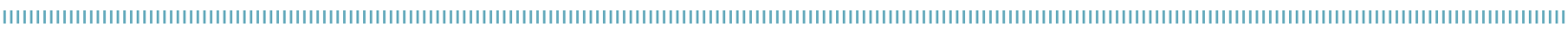

RESUMO: A encefalopatia espongiforme bovina (EEB), causada por um príon infectante, surgiu na década de 1980 na Europa como uma nova doença nos rebanhos bovinos e, desde então, estão sendo tomadas várias açôes para sua prevenção e controle. A restrição da alimentação de ruminantes com subprodutos de origem animal e a remoçáo e destruição dos materiais de risco específico para a doença das carcaças em frigoríficos se mostraram efetivas medidas para o controle da doença, além de reduzirem a exposição humana ao agente, pois se trata de uma importante zoonose. No entanto, em 2004 os primeiros casos atípicos de EEB foram diagnosticados, nos quais os agentes causais apresentavam alteraçóes de peso molecular na prova de Western blot, em relação ao agente da forma clássica. Além das diferenças moleculares dos agentes, as apresentações clínicas mostraram-se diferenciadas nas formas atípicas, acometendo principalmente bovinos com idade superior a oito anos. Por se tratar de uma nova forma da doença, muitos estudos estâo sendo conduzidos buscando elucidar a patogenia, epidemiologia e seu potencial zoonótico. Objetivou-se neste estudo revisar os principais aspectos relacionados às EEB atípicas enfatizando sua etiologia, epidemiologia, sinais clínicos, diagnóstico e medidas de controle.

PALAVRAS-CHAVE: príon; bovinos; $\mathrm{Pr}^{S C}$; EEB tipo L; EEB tipo H; EEB tipo SW.

\begin{abstract}
Bovine spongiform encephalopathy (BSE), caused by an infectious prion, emerged in the 1980s in Europe as a new disease in cattle and, since then, several actions are being taken for its prevention and control. Restricting the feeding of ruminants with animal by-products and the removal and destruction of specific risk materials (SRM) for the condition of carcasses in slaughterhouses have been proven effective to control the disease, in addition to the reduction of human exposure to the agent, as this is an important zoonosis. However, in 2004 the first atypical cases of BSE were diagnosed, in which the causative agents showed different molecular weights in Western blot (WB), compared to the classical form of the agent. In addition to the molecular differences, clinical presentations proved to be differentiated in atypical forms, affecting mainly cattle older than eight years. Because it is a new form of the disease, many studies are being conducted to elucidate the pathogenesis, epidemiology and zoonotic potential of atypical BSE. The aim of this study was to review the main aspects of atypical BSE emphasizing its etiology, epidemiology, clinical signs, diagnosis and control and prevention measures.
\end{abstract}

KEYWORDS: prion; cattle; $\mathrm{PrP}^{\mathrm{SC}}$; L-BSE; H-BSE; BSE-SW.

\footnotetext{
'Superintendência Federal de Agricultura no Paraná, Serviço de Saúde Animal, Ministério da Agricultura, Pecuária e Abastecimento - Curitiba (PR), Brasil. ${ }^{2}$ Departamento de Medicina Veterinária, Setor de Ciências Agrárias, Universidade Federal do Paraná - Curitiba (PR), Brasil.

*Autor correspondente: ellen.laurindo@agricultura.gov.br 


\section{INTRODUÇÃO}

A encefalopatia espongiforme bovina (EEB), comumente conhecida como "doença da vaca louca", é uma doença degenerativa fatal e transmissível do sistema nervoso central (SNC) de bovinos, com longo período de incubação (média de cinco anos), diagnosticada pela primeira vez em 1986 na Europa (ANDERSON et al., 1996; WELLS et al., 1987). Pertence ao grupo das encefalopatias espongiformes transmissíveis (EET), doenças causadas por um agente chamado príon $\left(\operatorname{Pr} \mathrm{P}^{S C}\right)$, uma proteína de conformação espacial alterada e com potencial infeccioso (PRUSINER et al., 1982). Estudos epidemiológicos desenvolvidos após o aparecimento dos primeiros casos de EEB apontaram a via oral como a principal forma de transmissão do agente, pela ingestáo de farinha de carne e ossos (FCO) contendo carcaças de animais positivos (WILESMITH et al., 1988). Os sinais clínicos mais característicos são as alterações comportamentais, a hipersensibilidade aos sons e toques e a apreensão (BRAUN et al., 1998), mas os sinais podem variar de acordo com a região cerebral afetada (SAEGERMAN et al., 2004).

O pico da epidemia da EEB na Europa foi no ano de 1992, quando foram registrados 37.316 casos da doença (OIE, 2015). Apesar dos esforços para evitar a entrada de tecidos bovinos potencialmente infectados na cadeia alimentar humana, em 1996 foi comprovada a transmissão da EEB a humanos, causando a variante da doença de Creutzfeldt-Jakob (vDCJ) (WILL et al., 1996), resultando em um impacto devastador na indústria pecuária do Reino Unido e no mundo (BROWN et al., 2001). Devido a esse grande impacto econômico e por ser uma zoonose, as autoridades sanitárias implantaram medidas para proteger a saúde humana e animal, que incluíram a proibição da alimentação de ruminantes com alguns subprodutos de origem animal, a proibição do consumo de carne e subprodutos de animais doentes, a remoção do material de risco específico (MRE) das carcaças de bovinos, vigilância epidemiológica e controle de subprodutos e importaçáo de animais vivos (SEUBERLICH et al., 2010). Devido a algumas características da doença, como o longo período de incubação, levaram-se mais de duas décadas para que ela fosse finalmente controlada. Contudo, apesar da EEB estar controlada, novas formas, chamadas de EEB atípicas, estão sendo diagnosticadas no mundo. Atualmente, o desafio é caracterizar essas formas atípicas, determinar se as medidas de controle da EEB clássica são efetivas para ambas as formas e mensurar seus possíveis impactos na saúde pública.

Até recentemente, estudos sobre a transmissão a partir de isolados de casos de EEB a campo e casos da vDCJ em seres humanos demonstraram apenas um agente causal (BRUCE et al., 1997). Além disso, todos os $\operatorname{PrP}^{S C}$ isolados do campo mostraram peso molecular e perfis de glicosilação semelhantes, utilizando técnicas de Western blot (WB) (GAVIER-WIDÉN et al., 2005). Por essas razóes, era amplamente aceita, pela comunidade científica, a existência de apenas uma forma de príon causador da EEB, ao contrário do que se observava na Scrapie desde a década de 1970 (FRASER; DICKINSON, 1973).

Os primeiros casos atípicos de EEB foram diagnosticados, quase que simultaneamente, na França e Itália, em 2004. O caso francês demonstrou a existência de uma porção náo glicosilada do $\operatorname{Pr}^{S C}$ com peso molecular mais alto do que o observado na EEB clássica, sendo então classificado como EEB tipo H (BIACABE et al., 2004). Em contraste, o peso molecular da porção não glicosilada do $\operatorname{Pr} \mathrm{P}^{S C}$ encontrado no caso italiano era menor do que o da EEB clássica, sendo classificado como EEB tipo L (CASALONE et al., 2004). No início de 2016 foi relatada a identificação de uma terceira forma de EEB atípica, classificada como EEB tipo SW, porém essa nunca foi observada em casos de campo (MASUJIN et al., 2016).

As duas primeiras formas receberam a denominaçáo $\mathrm{L}$ e $\mathrm{H}$ em referência a essa característica molecular encontrada no exame de WB, onde L refere-se a lower, ou seja, menor, e $\mathrm{H}$ (higher), ao maior peso molecular (BUSCHMANN et al., 2006). A terceira forma atípica, identificada em 2016, recebeu a denominaçáo $S W$ em referência às características clínicas observadas nos animais infectados experimentalmente, como curto período de incubação e perda de peso. Neste caso, $S$ refere-se a short incubation period (curto período de incubação) e W refere-se a weight loss (perda de peso) (MASUJIN et al., 2016).

$\mathrm{Na}$ EEB tipo L, exames de imunohistoquímica (IHQ) revelaram a presença de placas amiloidóticas, semelhantes às observadas em outras EET em seres humanos, como na doença de Creutzfeldt-Jacob (DCJ), na nova vCJD, na doença de Ger stmann-Sträussler-Scheinker (GSS) e Kuru, que, até então, nunca haviam sido reportadas em animais. Assim, os pesquisadores italianos também denominaram esse novo fenótipo de doença como encefalopatia espongiforme amiloidótica bovina (BASE) (CASALONE et al., 2004).

Apesar do primeiro caso atípico ter sido diagnosticado na França em 2004, um estudo retrospectivo, utilizando material de casos positivos diagnosticados nesse país desde 2001, revelou que o primeiro caso de EEB tipo $\mathrm{H}$ ocorreu, na verdade, no ano 2000 (BIACABE et al., 2008). Isso demonstra que as formas atípicas da EEB não são recentes, elas somente não foram devidamente diferenciadas em anos anteriores.

Da mesma forma que no tipo $\mathrm{H}$, há indícios que a $\mathrm{EEB}$ tipo L já tenha ocorrido em anos anteriores. Apesar dela ter sido oficialmente diagnosticada apenas em 2004, trabalhos anteriores já demonstravam a existência de placas amiloides em casos positivos de EEB nos anos de 1991 e 1995 (WELLS; WILESMITH, 1995), porém, a ausência de exames de WB inviabilizou a diferenciação do agente à época. Ainda não se têm estudos retrospectivos disponíveis relacionados à EEB tipo SW. 
A identificação de outras formas de EEB pode ser atribuída ao aumento da vigilância das EET em todo o mundo, especialmente na Europa (SEUBERLICH et al., 2010). A descoberta de formas atípicas da EEB em vários países sugere claramente que sua ocorrência não está restrita a uma regiáo geográfica (BUSCHMANN et al., 2006). Alguns casos atípicos diagnosticados no mundo estão descritos na Tabela 1.

Ambos os tipos de EEB já foram diagnosticados no mundo todo, em animais idosos, e sua baixa prevalência pode indicar que se trata de formas espontâneas da doença no rebanho (BIACABE et al., 2008). Porém, o alcance estatístico de estudos epidemiológicos envolvendo as formas atípicas de EEB ainda é limitado devido ao baixo número de casos e à escassez de informaçôes epidemiológicas sobre eles (SALA et al., 2012).
Uma das características das formas atípicas da EEB é a ocorrência em animais idosos (acima de oito anos de idade). $\mathrm{O}$ primeiro estudo sobre a epidemiologia das EEB atípicas analisou os casos franceses e demonstrou que a média de idade dos bovinos acometidos pelas formas atípicas (tipo $\mathrm{H} \mathrm{e} \mathrm{L}$ ) era de 12 anos (variando entre 7 e 18 anos para o tipo $\mathrm{L}$ e 8 a 19 anos para o tipo H), sendo significativamente maior do que a média de idade da EEB clássica (média de 7 anos, variando entre 3 e 15 anos) (SALA et al., 2012). Não houve diferença significativa entre as médias de idade de ocorrência das EEB tipos L e H.

Para muitos cientistas, a hipótese mais aceitável para a origem das EEB atípicas é a forma espontânea, podendo refletir um processo natural de envelhecimento e, talvez, tenha algumas características em comum com outras doenças

Tabela 1. Sumário dos casos de encefalopatia espongiforme bovina atípicas diagnosticados em bovinos.

\begin{tabular}{|c|c|c|c|c|}
\hline País & Idade & Sinais clínicos & $\begin{array}{l}\text { Caraterização no } \\
\text { Western blot }\end{array}$ & Referências \\
\hline \multirow{2}{*}{ Itália } & 11 & Não & $\mathrm{L}$ & \multirow{2}{*}{ CASALONE et al., 2004} \\
\hline & 15 & Não & $\mathrm{L}$ & \\
\hline Dinamarca & 14 & Não & $\mathrm{L}$ & DUCROT et al., 2008 \\
\hline Polônia & 12 & Não & $\mathrm{L}$ & POLAK et al., 2004 \\
\hline \multirow{2}{*}{ Japão } & 2 & Não & $L^{*}$ & YAMAKAWA et al., 2003 \\
\hline & 14 & Distasia & $\mathrm{H}$ & MASUJIN et al., 2008 \\
\hline Bélgica & 5,5 & Não & $L^{*}$ & De BOSSCHERE et al., 2004 \\
\hline \multirow{3}{*}{ França } & 10 & Não & $\mathrm{H}$ & \multirow{3}{*}{ BIACAGE et al., 2004} \\
\hline & 15 & Não & $\mathrm{H}$ & \\
\hline & 8 & Não & $\mathrm{H}$ & \\
\hline Holanda & 13 & NA & $\mathrm{H}$ & JACOBS et al., 2007 \\
\hline Suécia & 12 & Caído & $\mathrm{H}$ & GAVIER-WIDÉN et al., 2008 \\
\hline \multirow[t]{2}{*}{ Suiça } & 19 & $\begin{array}{l}\text { Típicos de encefalopatia } \\
\text { espongiforme bovina }\end{array}$ & $\mathrm{H}$ & SEUBERLICH et al., 2006 \\
\hline & 6,5 & Não & $\mathrm{H}$ & GULDIMANN et al., 2012 \\
\hline \multirow{2}{*}{ Alemanha } & 13 & Não informado & $\mathrm{H}$ & \multirow{2}{*}{ BUSCHMANN et al., 2006} \\
\hline & 15 & Não informado & $\mathrm{L}$ & \\
\hline \multirow{2}{*}{ Estados Unidos } & 12 & Caído & $\mathrm{H}$ & \multirow{2}{*}{ RICHT et al., 2007} \\
\hline & 10 & Caído & $\mathrm{H}$ & \\
\hline \multirow{7}{*}{ Reino Unido } & 13 & Ataxia & $\mathrm{L}$ & \multirow{4}{*}{ STACK et al., 2009} \\
\hline & 11 & Não & $\mathrm{L}$ & \\
\hline & 12 & Não & $\mathrm{L}$ & \\
\hline & 21 & Não & $\mathrm{L}$ & \\
\hline & 13 & Caído & $\mathrm{H}$ & TERRY et al., 2007 \\
\hline & 14 & Caído/paresia & $\mathrm{H}$ & \multirow{2}{*}{ STACK et al., 2009} \\
\hline & 15 & Caído & $\mathrm{H}$ & \\
\hline Canadá & 16 & Caído & $\mathrm{H}$ & DUDAS et al., 2010 \\
\hline \multirow{2}{*}{ Brasil } & 13 & Caído & $\mathrm{H}$ & OIE, 2015 \\
\hline & 12 & Não & $\mathrm{H}$ & OIE, 2015 \\
\hline Noruega & 15 & Não & $\mathrm{H}$ & OIE, 2015 \\
\hline Portugal & 13 & Caído & $\mathrm{H}$ & ORGE et al., 2015 \\
\hline Romênia & Não Informado & Não informado & $\mathrm{L}$ & BARBUCEANU et al., 2015 \\
\hline Eslovênia & 12 & Não informado & $\mathrm{H}$ & OIE, 2015 \\
\hline
\end{tabular}

*Não estão classificados no meio científico (DUDAS et al., 2010). 
neurodegenerativas em humanos, como o mal de Alzheimer (CISSE; MUCKE, 2009). Essa hipótese é reforçada pela idade avançada dos animais acometidos, sua distribuição geográfica heterogênea e pela ausência de ligaçóes epidemiológicas com outras EET (SEUBERLICH et al., 2010).

Apesar dessas afirmaçóes sobre a origem das EEB atípicas, alguns autores consideram prematuro atribuir a essas doenças uma origem espontânea, até que se tenham mais estudos sobre as formas de transmissão (SEUBERLICH et al., 2010).

Em vários casos de $\mathrm{EEB}$ tipos $\mathrm{L}$ e $\mathrm{H}$, houve o sequenciamento do gene PrP buscando identificar alguma mutação que pudesse dar origem à doença. Mas, com exceção de um caso detectado nos Estados Unidos (RICHT et al., 2007), que apresentava uma mutação responsável por codificar lisina no lugar do ácido glutâmico no códon 211 (primeiro caso de EET de origem genética em bovinos), não foram observadas diferenças dos casos clássicos (BRUNELLE et al., 2007; BUSCHMANN et al., 2006; CLAWSON et al., 2008). Dessa forma, pode-se praticamente descartar a hipótese de origem genética das EEB atípicas (BUSCHMANN et al., 2006).

O estudo pioneiro sobre a epidemiologia das EEB atípicas foi realizado em 2012, na França, e demonstrou que, ao contrário da EEB clássica, as formas atípicas eram mais frequentes em bovinos de aptidáo corte quando comparados com os de aptidáo leite (81,8\% dos casos de EEB tipo $\mathrm{H}$ e $83,3 \%$ de EEB tipo $\mathrm{L}$ foram diagnosticados em bovinos de corte, contra 20,3\% de casos de EEB clássica nessa mesma categoria) (SALA et al., 2012).

Ainda na França, pesquisas demonstraram que a incidência de EEB tipo $\mathrm{L}$ e tipo $\mathrm{H}$ foram constantes ao longo dos anos, e que não estão relacionadas aos casos clássicos da doença (BIACAGE et al., 2008).

De qualquer forma, independente da determinação de sua prevalência ou incidência, o fato das EEB atípicas não estarem relacionadas aos casos clássicos da doença reforça a hipótese que essas representam formas esporádicas de EET, porém, não é possível nesse momento excluir outras origens (SEUBERLICH et al., 2010).

As pesquisas sobre a neuropatologia e distribuição do $\mathrm{PrP}^{\mathrm{SC}}$ da EEB, tanto do tipo $\mathrm{H}$ quanto do $\mathrm{L}$, são limitadas (SEUBERLICH et al., 2010). Isso se deve ao fato de não se ter encéfalos e/ou carcaças inteiras oriundas de bovinos positivos disponíveis para estudo pois, na vigilância de rotina (onde normalmente se detecta esses casos), somente a regiáo do óbex é coletada para análise, sendo que as demais partes do encéfalo, bem como a carcaça, acabam sendo destruídas (OKADA et al., 2011). Além da escassez de material para pesquisas, muitos casos atípicos são oriundos de animais caídos e, normalmente, o tecido coletado desses indivíduos já está em algum estado de decomposição, comprometendo ainda mais estudos sobre patogenia e transmissibilidade (KONOLD et al., 2012).

Considerando essas limitaçóes, todo o conhecimento que se tem atualmente sobre transmissão e patogenia das EEB atípicas baseia-se em experimentos realizados por inoculaçôes experimentais em camundongos, primatas não humanos $\mathrm{e}$ bovinos, utilizando inóculos a partir de material positivo de bovinos diagnosticados durante as açôes de vigilância para a EEB. A maioria dos experimentos de transmissibilidade utiliza inoculaçóes pelas vias intracerebral e intraperitoneal (pela reduzida quantidade de material biológico positivo), não refletindo as reais barreiras interespecíficas observadas numa infecção oral (SEUBERLICH et al., 2010).

Apesar da infecção por via oral não ser capaz de definir a virulência dos agentes das EEB (clássica, tipo $\mathrm{H}$ ou tipo $\mathrm{L}$ ) (MANUELIDIS, 2010), seu conhecimento é importante para a definiçáo das medidas de controle da doença. Dessa forma, várias linhas de pesquisa sobre a transmissibilidade das EEB atípicas estão trabalhando com desafios orais, considerando que esta é a via mais importante de transmissão do agente e, consequentemente, seu conhecimento é necessário para a prevenção de futuras epidemias. Devido ao longo período de incubaçáo da EEB, há poucos estudos concluídos sobre transmissão oral dessas formas (SEUBERLICH et al., 2010).

Estudos já demonstraram que, assim como o príon causador da EEB clássica, os agentes causadores das EEB tipos L e $\mathrm{H}$ também conseguem infectar outras espécies (BÉRINGUE et al., 2007; BARON et al., 2006).

Sobre a patogenia das EEB atípicas, os primeiros relatos dessas doenças já demonstraram que a distribuição do $\mathrm{Pr} \mathrm{P}^{\mathrm{SC}}$ em diferentes regiôes do cérebro não seguiam o padrão determinado nos casos de EEB clássica (CASALONE et al., 2004; BIACAGE et al., 2004).

O primeiro estudo sobre a patogenia das EEB atípicas foi desenvolvido em 2011, com desafios utilizando os agentes das EEB tipos $\mathrm{L}$ e $\mathrm{H}$ em bovinos por via intracerebral (BALKEMA-BUSCHMANN et al., 2011a). Não se verificaram diferenças na distribuição do $\operatorname{PrP}^{S C}$ das formas atípicas em relaçáo à EEB clássica, sendo mínima a concentração em todos os tecidos periféricos analisados. Outra pesquisa também não detectou a presença do agente em nenhum tecido linfoide ou tecido nervoso entérico dos animais desafiados com EEB tipos L e H (KONOLD et al., 2012).

Em relação ao $\mathrm{SNC}$, foram observadas alteraçóes na distribuição do agente da EEB tipos L e H em relação à forma clássica apenas no encéfalo, em desafios intracerebrais, havendo maior vacuolização nas regiôes rostrais do encéfalo, especialmente no córtex frontal (KONOLD et al., 2012). Apesar da distribuição mais rostral desses agentes no $\mathrm{SNC}$, ambos foram detectados no óbex de todos os animais desafiados.

\section{Encefalopatia espongiforme bovina tipo $L$}

A EEB tipo L só conseguiu ser transmitida a camundongos comuns após a segunda passagem, ao contrário do observado com os tipos He clássico (CAPOBIANCO et al., 2007), porém, 
desenvolveram sinais clínicos após um período médio de 185 dias de incubação, enquanto o período de incubação foi de 230 dias na clássica (BUSCHMANN et al., 2006). Nesses animais, o $\mathrm{PrP}^{\mathrm{PC}}$ da EEB tipo L adquire características moleculares e fenótipos neuropatológicos semelhantes à EEB clássica (BÉRINGUE et al., 2006), podendo ter uma importante implicaçáo em relação à origem da EEB em bovinos e das EET em outras espécies, incluindo seres humanos (CAPOBIANCO et al., 2007). Apesar desses achados em camundongos, não se observou a conversão do príon em sua forma clássica em inoculaçôes intracerebrais em bovinos (IWAMARU et al., 2010; BALKEMA-BUSCHMANN et al., 2011b; KONOLD et al., 2012), mesmo após uma segunda passagem (KONOLD et al., 2014).

Outro estudo demonstrou um reduzido envolvimento do núcleo motor dorsal do nervo vago e do tronco encefálico na patogenia da EEB tipo $\mathrm{L}$ em bovinos, sugerindo que a rota de disseminaçáo do agente náo se dá pelo trato digestório, podendo indicar que se trata de uma doença de ocorrência esporádica no rebanho (CASALONE et al., 2004).

$\mathrm{O}$ agente da EEB tipo $\mathrm{L}$ pode ser transmitido a camundongos Tg650 PrP humano, sem barreira interespecífica significante, pois experimentalmente houve $100 \%$ de taxa de ataque, além de não haver alteraçóes no perfil eletroforético do PrP após seguidas passagens (BÉRINGUE et al., 2008). Além disso, outro experimento demonstrou que esse agente tem tropismo por tecidos linfoides quando inoculado por via intracerebral em camundongos transgênicos para $\operatorname{PrP}^{\mathrm{C}}$ humana (KONG et al., 2008).

Estudos posteriores demonstraram que não houve depósito de $\mathrm{PrP}^{\mathrm{SC}}$ da EEB tipo L nos tecidos linfoides e linfonodos dos macacos inoculados com esse agente (ONO et al., 2011), porém, o desenvolvimento dos sinais clínicos nesses animais foi mais precoce (21 meses) quando comparados aos desafiados com a forma clássica e com a vDCJ, com tempo de sobrevida significativamente menor (COMOY et al., 2008).

Em resumo, todos os experimentos de transmissão da EEB tipo L para camundongos transgênicos humanos (KONG et al., 2008) e primatas (COMOY et al., 2008; ONO et al., 2011) revelaram que esse agente é muito mais virulento nessas espécies do que se observa na forma clássica da EEB. Essa característica é uma forte indicação de um alto potencial zoonótico da EEB tipo L, em relação à EEB clássica (BALKEMA-BUSCHMANN et al., 2011a). No entanto, é necessária a realização de experimentos utilizando a inoculação do agente da EEB tipo L por via oral para uma melhor avaliação da transmissão do agente para seres humanos a partir de produtos de ruminantes infectados (ONO et al., 2011).

Em bovinos, estudos demonstraram que não houve transformação do príon da EEB tipo L para a sua forma clássica (EEB clássica) e que o período de incubação da doença atípica foi significativamente menor. Não houve detecção do $\operatorname{PrP}^{\mathrm{SC}}$ nos tecidos linfoides de nenhum animal desafiado (forma clássica e tipo L), porém, observou-se atrofia muscular nos músculos glúteo médio, psoas maior, longuissimus dorsis e tríceps (LOMBARDI et al., 2008). Já outro trabalho demonstrou que o período de incubação de ambas as formas atípicas é mais prolongado, quando comparado a forma clássica da EEB em bovinos, o que justifica a ocorrência dessas formas em animais mais velhos (acima de oito anos) (BALKEMA-BUSCHMANN et al., 2011b).

Em inoculações intracerebrais em bovinos, o agente da EEB tipo L se propaga, inicialmente, no SNC e, da mesma forma que o observado na forma clássica (HOFFMANN et al., 2007), na fase terminal da doença há uma difusão centrífuga pelas terminaçóes nervosas a outros tecidos nervosos (IWAMARU et al., 2010). O conhecimento sobre a difusão do agente da EEB tipo $L$ nos tecidos dos bovinos é importante para que haja a correta definição dos MRE a serem removidos e destruídos, garantindo a proteçáo dos consumidores (IWAMARU et al., 2010).

\section{Encefalopatia espongiforme bovina tipo $\mathrm{H}$}

$\mathrm{O}$ agente da $\mathrm{EEB}$ tipo $\mathrm{H}$ foi transmitido experimentalmente em camundongos normais (BARON et al., 2006; CAPOBIANCO et al., 2007) e em camundongos transgênicos PrP bovino e ovino (BÉRINGUE et al., 2006; 2007). Nesses experimentos não houve conversão do agente ou alteraçấo fenotípica para o tipo clássico, como se observou na EEB tipo L. Porém, estudos posteriores demonstraram que o agente da EEB tipo $\mathrm{H}$ foi capaz de se converter no tipo clássico após sucessivas passagens em camundongos comuns (BARON et al., 2011); e em camundongos transgênicos para $\mathrm{PrP}^{\mathrm{C}}$ bovino (TgBov) (TORRES et al., 2011). Esses resultados demonstram a capacidade do príon da EEB tipo $\mathrm{H}$ adquirir características da forma clássica, quando da presença de proteína $\mathrm{PrP}^{\mathrm{C}}$ bovina. Isso reforça a hipótese de que o agente que originou a epidemia de EEB clássica pode ter se originado a partir dessa forma atípica (TORRES et al., 2011).

Em inoculações intracerebrais em camundongos transgênicos tg650 $\operatorname{PrP}$ humano, $\mathrm{o}$ isolado de EEB tipo $\mathrm{H}$ não infectou nem transmitiu a doença a esses animais. Esse resultado reforça a hipótese de que há uma forte barreira de transmissão desse tipo de EEB entre os bovinos e seres humanos (BÉRINGUE et al., 2008).

Após sucessivas passagens do agente da EEB tipo $\mathrm{H}$ em cérebros de camundongos transgênicos $\mathrm{TgBov}$ e posterior inoculação intracerebral em bovinos, um estudo demonstrou a alteraçáo do príon para a forma de EEB tipo SW. Nesse caso, o período de incubaçáo, tanto nos camundongos transgênicos como nos bovinos, foi menor do que o observado nas formas clássica, tipo $\mathrm{L}$ e tipo $\mathrm{H}$, indicando maior virulência dessa nova forma (MASUJIN et al., 2016).

Em inoculaçáo intracerebral em bovinos, estudos demonstraram que o agente da EEB tipo $\mathrm{H}$ teve distribuição mais cortical (córtex frontal, tálamo e hipotálamo) e com escore de lesão vacuolar superior ao observado nos casos de EEB clássica (OKADA et al., 2011). 


\section{SINAIS CLÍNICOS}

De forma geral, as EEB atípicas, ao contrário da forma clássica não estão sendo diagnosticadas em animais com sinais clínicos neurológicos, mas sim em bovinos aparentemente saudáveis encaminhados ao abate de rotina, ao abate de emergência ou em bovinos em decúbito, sugerindo que sua apresentação clínica é diferente da EEB clássica (KONOLD et al., 2012).

Apesar de estudos demonstrarem não haver diferença estatística entre a síndrome nervosa observada em bovinos inoculados com as formas atípicas e clássicas de EEB, há diferenças notáveis em relação à forma clássica: a ausência de tremores, a dificuldade de levantar e a dismetria já nas fases iniciais da doença, em todas as formas atípicas (KONOLD et al., 2012).

Em relaçáo aos sinais clínicos, postulou-se que as forma clássica e atípicas de EEB em bovinos parecem produzir dois fenótipos clínicos principais: a forma nervosa ou a forma "apática”, sendo a EEB clássica a que apresenta os sinais clínicos mais intensos e uniformes (especialmente em relação ao nervosismo) (KONOLD et al., 2012). O desenvolvimento da forma apática só foi observado em bovinos com curso clínico prolongado, e que previamente apresentaram a forma nervosa da doença. Isso pode sugerir que todos os bovinos acometidos com as formas atípicas da EEB podem eventualmente desenvolver a forma apática, caso não sejam sacrificados ou abatidos antes (KONOLD et al., 2012).

Nos estudos realizados até o presente momento, todos os bovinos inoculados com as EEB atípicas apresentaram dificuldades para levantar (BALKEMA-BUSCHMANN et al., 2011b; KONOLD et al., 2012). Essa característica clínica explica a razão pela qual a maior parte dos casos de EEB atípicas diagnosticados a campo estarem relacionados a animais caídos (RICHT et al., 2007; STACK et al., 2009; GAVIER-WIDÉN et al., 2008).

A duração dos sinais clínicos das formas atípicas de EEB foi de 4 a 10 meses para a EEB tipo L, e 2 a 7 meses para a EEB tipo $\mathrm{H}$, (KONOLD et al., 2012) sendo mais longa quando comparada a outros estudos (LOMBARDI et al., 2008; FUKUDA et al., 2009) e menor quando comparada ao estudo de OKADA et al. (2011). Essas variações na duração clínica da doença podem ser atribuídas às raças dos bovinos utilizadas nesses estudos (KONOLD et al., 2012), da mesma forma que ocorreu com estudos da forma clássica da EEB (WILESMITH et al., 1992).

Observa-se que ainda não é possível definir claramente quais os sinais clínicos característicos das formas atípicas, bem como seu período de incubação e tempo de manifestação clínica, pois poucos estudos chegam à mesma conclusão.

\section{DIAGNÓSTICO}

Até 2004, pensava-se que a conformação no WB era única para todos os $\mathrm{PrP}^{\mathrm{SC}}$ tanto na $\mathrm{EEB}$ natural quanto na experimental
(STACK, 2004). Estudos posteriores demonstraram a existência de outras conformações no WB, relacionadas a formas atípicas de EEB, o que criou um desafio no campo diagnóstico.

O diagnóstico clínico das formas atípicas de EEB é bastante limitado, pois ainda estão sendo definidos quais sinais são característicos dessas enfermidades (SEUBERLICH et al., 2010). Outro estudo concluiu ser impossível o diagnóstico das formas atípicas apenas com os achados clínicos (BALKEMA-BUSCHMANN et al., 2011b).

Com base nos estudos de sinais clínicos já realizados, pode-se considerar as EEB atípicas em diagnósticos clínicos diferenciais em bovinos idosos (com mais de oito anos) encontrados caídos, com dificuldade de se levantar e com histórico ou presença de reaçôes exacerbadas a estímulos externos (KONOLD et al., 2012).

Considerando que estudos de patogenia e distribuição do agente das EEB atípicas demonstraram que os depósitos do $\mathrm{PrP}^{\mathrm{SC}}$ estão distribuídos nas porçôes mais craniais do encéfalo e também no cerebelo, pode ser considerada a possibilidade de se ampliar os materiais de eleiçâo para diagnóstico nas açôes de vigilância ativa de EEB, incluindo o cerebelo (que pode ser retirado, da mesma forma que o tronco encefálico, pelo forame magno, sem a necessidade da abertura da cabeça) (KONOLD et al., 2012).

O diagnóstico diferencial das atípicas baseia-se nas características moleculares do PrP ${ }^{\mathrm{RES}}$ identificadas através da técnica de WB (MELONI et al., 2012). Após a digestão pela proteinase $\mathrm{K}$, o $\operatorname{PrPRES}$ pode apresentar três glicoformas: a náo glicosilada, a monoglicosilada e a diglicosilada. Dependendo da quantidade de cada uma dessas glicoformas e sua posiçáo na banda do WB, faz-se a separação dos agentes da EEB nos tipos clássico, H (higher) e L (lower) (JACOBS et al., 2007) e SW (MASUJIN et al., 2016).

As EEB tipos $\mathrm{H}$ e $\mathrm{L}$ apresentam massa molecular maior e menor, respectivamente, nas porçôes não glicosiladas do $\operatorname{PrP}^{\mathrm{RES}}$ pela técnica de WB em relação à forma clássica. Já a forma $\mathrm{SW}$ é mais leve do que a forma $\mathrm{H}$ e mais semelhante à clássica (MASUJIN et al., 2016). Além disso, a EEB tipo L tem uma menor proporção de $\mathrm{PrP}^{\mathrm{RES}}$ diglicosilado, sendo a característica molecular mais marcante desse agente (CASALONE et al., 2004).

\section{MEDIDAS DE CONTROLE}

Desde 2004, alguns autores reforçam a possibilidade da EEB ter diferentes manifestaçôes, o que poderia impactar nas medidas de controle da doença em vigor já naquela época. Além disso, reiteraram a necessidade da realização de novos estudos para determinar a frequência desses príons atípicos na população bovina e outros achados biológicos envolvendo essa cepa infectante (BIACAGE et al., 2004).

Considerando uma possível origem espontânea e esporádica das formas atípicas, é factível que elas persistam nos 
rebanhos bovinos mesmo após a erradicaçáo da forma clássica da EEB. Considerando que tanto o agente da EEB tipo L (BÉRINGUE et al., 2006) quanto o tipo H (GULDIMANN et al., 2012) se converteram na forma clássica após passagens em camundongos, há um risco constante da reemergência da EEB clássica caso a proibiçáo do uso de subprodutos de origem animal na alimentaçấo de ruminantes (feed ban) seja diminuída ou revogada.

Apesar da ausência de estudos que esclareçam a manifestação e patogenia da EEB tipo SW, a manutenção das açóes de prevenção da EEB, como o feedban, é necessária mesmo após a erradicação da EEB clássica (MASUJIN et al., 2016). Assim, algumas medidas de mitigaçáo de risco deverão ser continuamente e permanentemente mantidas, até que se tenha maior conhecimento sobre o potencial infeccioso e zoonótico das EEB atípicas (GULDIMANN et al., 2012).

O conhecimento sobre a patogenia das formas atípicas da EEB é fundamental para a determinação das medidas de controle da doença, em especial o potencial risco da exposição humana ao agente. Atualmente, assume-se que a distribuição do agente das atípicas, no SNC, é semelhante ao observado nos casos clássicos. Porém, se for comprovado que essa distribuição é diferenciada, principalmente no que se refere aos MRE, poderemos ter problemas de saúde pública e na mitigação de risco (SEUBERLICH et al., 2010).

É interessante ressaltar que o número de casos de EEB atípicas é infinitamente inferior aos casos clássicos durante a epidemia nos anos 1990. Por essa razão a demonstração do possível caráter zoonótico das EEB atípicas pode não acarretar na pronta mudança nas açóes de mitigaçáo de risco dessa doença, pois o risco de exposição a esses agentes é baixo (SEUBERLICH et al., 2010).

Embora já se tenha confirmado a ligação entre a EEB clássica e a vCJD em seres humanos (WILL et al., 1996), não foi identificada nenhuma associação entre as EEB atípicas e as EET humanas: não há ligação epidemiológica que indique que se trata de zoonoses. Porém, as características moleculares da EEB tipo L são semelhantes à DCJ, além disso, os estudos utilizando camundongos transgênicos de PrP humano e primatas demonstraram que o agente da EEB tipo L se replica com mais eficiência do que o da EEB clássica (BÉRINGUE et al., 2008; COMOY et al., 2008; KONG et al., 2008).

BROWN et al. (2006) ponderaram que existe a possibilidade de que ao menos alguns casos de DCJ esporádica possam ser relacionados à infecçáo humana pelo consumo de animais infectados EEB atípicas, porém, não há ligações epidemiológicas visto que não há comprovação, em nenhum dos casos, de exposição a animais infectados. Atribui-se a dificuldade de se estabelecer ligaçôes entre a EEB atípica e a DCJ ao pouco conhecimento sobre os parâmetros de infecçáo das formas atípicas da EEB, como por exemplo, hospedeiro, infectividade em tecidos, rota de transmissão e dose mínima infectante para seres humanos.

Considerando que há uma pressão para a retirada da proibição de alimentar ruminantes com FCO, esses aspectos devem ser fortemente considerados para evitar o risco de uma reemergência da EEB nos rebanhos (SEUBERLICH et al., 2010).

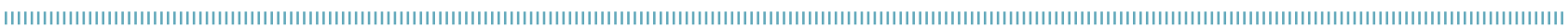
REFERÊNCIAS

ANDERSON, R.M.; DONNELLY, C.A.; FERGUSON, N.M.; WOOLHOUSE, M.E.; WATT, C.J.; UDY, H.J.; MAWHINNEY, S.; DUNSTAN, S.P.; SOUTHWOOD, T.R.; WILESMITH, J.W.; RYAN, J.B.; HOINVILLE, L.J.; HILLERTON, J.E.; AUSTIN, A.R.; WELLS, G.A. Transmission dynamics and epidemiology of BSE in British cattle. Nature, v.382, p.779-788, 1996.

BALKEMA-BUSCHMANN, A.; FAST, C.; KAATZ, M.; EIDEN, M.; ZIEGLER, U.; MCINTYRE, L.; KELLER, M.; HILLS, B.; GROSCHUP, M.H. Pathogenesis of classical and atypical BSE in cattle. Preventive Veterinary Medicine, v. 102, p.112-117, $2011 \mathrm{a}$.

BALKEMA-BUSCHMANN, A.; ZIEGLER, U.; MCINTYRE, L.; KELLER, M.; HOFFMANN, C.; ROGERS, R.; HILLS, B.; GROSCHUP, M.H. Experimental challenge of cattle with german atypical BSE isolates. Journal of Toxicology and Environmental Health, v.74, p.103-109, 201 1b.

BARBUCEANU, F.; SIMMONS, M.; CHAPLIN, M.; DIACONU, C.; NICOLAE, S.; RAITA, S.; BELU, C.; PREDOI, G. Conduct of diagnosis in the case of occurrence of bovine spongiform encephalopathy in Romania. Prion, v.9, p. 12, 2015.
BARON, T.; VULIN, J.; BIACABE, A.G.; LAKHDAR, L.; VERCHERE, J.; TORRES, J.M.; BENCSIK, A. Emergence of classical BSE strain properties during serial passages of H-BSE in wild type mice. PLOS ONE, v.6, e 15839, 2011.

BARON, T.G.M.; BIACABE, A.G.; BENCSIK, A.; LANGEVELD, P.M. Transmission of new bovine prion to mice. Emerging Infectious Diseases, v.12, n.7, p.1125-1128, 2006.

BÉRINGUE, V.; ANDRÉOLETTI, O.; LEDUR, A.; ESSALMANI, R.; VILOTTE, J.L.; LACROUX, C.; REINE, F.; HERZOG, L.; BIACABE, A.G.; BARON, T.; CARAMELLI, M.; CASALONE, C.; LAUDE, H. A bovine prion acquires an epidemic bovine spongiform encephalopathy strain-like phenotype on interspecies transmission. The Journal of Neuroscience, v.27, p.6965-6971, 2007.

BÉRINGUE, V.; BENCSIK, A.; LE DUR, A.; REINE, F.; LAÏ, T.L.; CHENAIS, N.; TILLY, G.; BIACABE, A.G.; BARON, T.; VILOTTE, J.L.; LAUDE, $H$. Isolation from cattle of a prion strain distinct from that causing bovine spongiform encephalopathy. PLoS Pathogens, v.2, e $112,2006$. 
BÉRINGUE, V.; HERZOG, L.; REINE, F.; LEDUR, A.; CASALONE, C.; VILOTTE, J.L.; LAUDE, H. Transmission of atypical bovine prions to mice transgenic for human prion protein. Emerging Infectious Diseases, v. 14, n.12, p.1898-1901, 2008.

BIACABE, A.G.; LAPLANCHE, J.L.; RYDER, S.; BARON, T. Distinct molecular phenotypes in bovine prion diseases. EMBO Rep, v.5, n. 1, p. $110-115,2004$.

BIACABE, A.G.; MORIGNAT, E.; VULIN, J.; CALAVAS, D.; BARON, T. Atypical bovine spongiform encephalopathies, France, 2001-2007. Emerging Infectious Diseases, v.14, n.2, p.298-300, 2008.

BRAUN, U.; PUSTERLA, N.; SCHICKER, E. Bovine spongiform encephalopathy: diagnostic approach and clinical findings. Compendium on Continuing Education for the Practicing Veterinarian, v.20, p.270-278, 1998.

BROWN, P.; MCSHANE, L.M.; ZANUSSO, G.; DETWILER, L. On the question of sporadic or atypical bovine spongiform encephalopathy and Creutzfeldt-Jakob disease. Emerging Infectious Diseases, v.12, p.1816-1821, 2006.

BROWN, P.; WILL, R.G.; BRADLEY, R.; ASHER, D.M.; DETWILER, L. Bovine spongiform encephalopathy and variant of Creutzfeldt-Jakob disease: background, evolution, and current concerns. Emerging Infectious Diseases, v.7, p.6-16, 2001.

BRUCE, M.E.; WILL, R.G.; IRONSIDE, J.W.; MCCONNEL, I.; DRUMMOND, D.; SUTTIE, A.; MCCARDLE, L.; CHREE, A.; HOPE, J.; BIRKETT, C.; COUSENS, S.; FRASER, H.; BOSTOCK, C.J. Transmissions to mice indicate that "new variant" CJD is caused by BSE agent. Nature, v.389, p.498-501, 1997.

BRUNELLE, B.W.; HAMIR, A.N.; BARON, T.; BIACABE, A.G.; RICHT, J.A.; KUNKLE, R.A.; CUTLIP, R.C.; MILLER, J.M.; NICHOLSON, E.M. Polymorphisms of the prion gene promoter region that influence classical bovine spongiform encephalopathy susceptibility are not applicable to other transmissible spongiform encephalopathies in cattle. Journal of Animal Science, v.85, n. 12, p.3142-3147, 2007.

BUSCHMANN, A.; GRETZSCHEL, A.; BIACABE, A.G.; SCHIEBEL, K.; CORONA, C.; HOFFMANN, C.; EIDEN, M.; BARON, T.; CASALONE, C.; GROSCHUP, M.H. Atypical BSE in German - Proof of transmissibility and biochemical characterization. Veterinary Microbiology, v. 117 , p. 103-116, 2006.

CAPOBIANCO, R.; CASALONE, C.; SUARDI, S.; MANGIERI, M.; MICCOLO, C.; LIMIDO, L.; CATANIA, M.; ROSSI, G.; DI FEDE, G.; GIACONNE, G.; BRUZZONE, M.G.; MINATI, L.; CORONA, C.; ACUTIS, P.; GELMETTI, D.; LOMBARDI, G.; GROSCHUP, M.H.; BUSCHMANN, A.; ZANUSSO, G.; MONACO, S.; CARAMELLI, M.; TAGLIAVINI, F. Conversion of the BASE prion protein into the BSE strain: the origin of BSE? PLoS Pathogens, v. 3, p. 1-8, 2007.

CASALONE, C.; ZANUSSO, G.; ACUTIS, P.; FERRARI, S.; CAPUCCI, L.; TAGLIAVINI, F.; MONACO, S.; CARAMELLI, M. Identification of a second bovine amyloidotic spongiform encephalopathy: molecular similarities with sporadic Creutzfeldt-Jakob disease. Proceedings of the National Academy of Sciences of the United States of America, v.101, p.3065-3070, 2004.

CISSE, M.; MUCKE, L. Alzheimer's disease: a prion protein connection. Nature, v.457, p.1090-1091, 2009.
CLAWSON, M.L.; RICHT, J.A.; BARON, T.; BIACABE, A.G.; CZUB, S.; HEATON, M.P.; SMITH, T.P.L.; LAEGREID, W.W. Association of a bovine prion gene haplotype with atypical BSE. PLOSONE, v.3, e 1830, 2008.

COMOY, E.E.; CASALONE, C.; ETCHEGARAY, N.L.; ZANUSSO, G.; FREIRE, S.; MARCE, D.; AUVRE, F.; RUCHOUX, M.M.; FERRARI, S.; MONACO, S.; SALES, N.; CARAMELLI, M.; LEBOULCH, P.; BROWN, P.; LASMÉZAS, C.I.; DESLYS, J.P. Atypical BSE (BASE) transmitted from asymptomatic aging cattle to a primate. PLoSONE, v.3, e3017, 2008.

DE BOSSCHERE, H.; ROELS, S.; VANOPDENBOSCH, E. Atypical case of bovine spongiform encephalopathy in East-Flemish cow in Belgium. The International Journal of Applied Research in Veterinary Medicine, v.7, n.3, p. 1-4, 2004.

DUCROT, C.; ARNOLD, M.; DE KOEIJER, A.; HEIM, D.; CALAVAS, D. Review on the epidemiology and dynamics of BSE epidemics. Veterinary Research, v.39, p. 15-33, 2008.

DUDAS, S.; YANG, J.; GRAHAM, C.; CZUB, M.; MCALLISTER, T.A.; COULTHART, M.B.; CZUB, S. Molecular, biochemical and genetic characteristics of BSE in Canada. PLoS ONE, v.5, n.5, p. 1-8, 2010.

FRASER, H.; DICKINSON, A.G. Scrapie in mice: agent strain differences in the distribution and intensity of grey matter vacuolation. Journal of Comparative Pathology, v.83, p.29-40, 1973.

FUKUDA, S.; IWAMARU, Y.; IMAMURA, M.; MASUJIN, K.; SHIMIZU, Y.; MATSUURA, Y.; SHU, Y.; KURACHI, M.; KASAI, K.; MURAYAMA, Y.; ONOE, S.; HAGIWARA, K.; SATA, T.; MOHRI, S.; YOKOAMA, T.; OKADA, $H$. Intraspecies transmission of L-type-like bovine spongiform encephalopathy detected in Japan. Microbiology and Immunology, v.53, p.704-707, 2009.

GAVIER-WIDÉN, D.; NÖREMARK, M.; LANGEVELD, J.P.M.; STACK, M.; BIACABE, A.G.; VULIN, J.; CHAPLIN, M.; RICHT, J.A.; JACOBS, J.; ACIIN, A.; MONLEÓN, E.; RENSTRÖM, L.; KLINGEBORN, B.; BARON, T.G.M. Bovine spongiform encephalopathy in Sweden: an H-type variant. Journal of Veterinary Diagnostic Investigation, v.20, p.2-10, 2008.

GAVIER-WIDÉN, D.; STACK, M.J.; BARON, T.; BALACHANDRAN, A.; SIMMONS, M.M. Diagnosis of transmissible spongiform encephalopathies in animals: a review. Journal of Veterinary Diagnostic Investigation, v. 17, p.509-527, 2005.

GULDIMANN, C.; GSPONER, M.; DROGEMULLER, C.; OEVERMANN, A.; SEUBERLICH, T. Atypical H-type bovine spongiform encephalopathy in a cow born after the reinforced feed ban on meat-and-bone meal in Europe. Journal of Clinical Microbiology, v.50, p.4171-4174, 2012 .

HOFFMANN, C.; ZIEGLER, U.; BUSCHMANN, A.; WEBER, A.; KUPFER, L.; OELSCHLEGEL, A.; HAMMERSCHMIDT, B.; GROSCHUP, M.H. Prions spread via the autonomic nervous system from the gut to the central nervous system in cattle incubating bovine spongiform encephalopathy. Journal of General Virology, v.88, p.1048-1055, 2007.

IWAMARU, Y.; IMAMURA, M.; MATSUURA, Y.; MASUJIN, K.; SHIMIZU, Y.; SHU, Y.; KURACHI, M.; KASAI, K.; MURAYAMA, Y.; FUKUDA, S.; ONOE, S.; HAGIWARA, K.; YAMAKAWA, Y.; SATA, T.; MOHRI, S.; OKADA, H.; YOKOYAMA, T. Accumulation of L-type bovine prions in peripheral nerve tissues. Emerging Infectious Diseases, v.16, n.7, p.1151-1154, 2010. 
JACOBS, J.G.; LANGERVELD, J.P.; BIACABE, A.G.; ACUTIS, P.L.; POLAK, M.P.; GAVIER-WIDÉN, D.; BUSCHMANN, A.; CARAMELLI, A.; CASALONE, C.; MAZZA, M.; GROSCHUP, M.; ERKENS, J.H.F.; DAVIDSE, A.; VAN ZIJDERVELD, F.G.; BARON, T. Molecular discrimination of atypical bovine spongiform encephalopathy strains from a geographical region spanning a wide area in Europe. Journal of Clinical Microbiology, v.45, p.1821-1829, 2007.

KONG, Q.; ZHENG, M.; CASALONE, C.; QING, L.; HUANG, S.; CHAKRABORTY, B.; WANG, P.; CHEN, F.; CALI, I.; CORONA, C.; MARTUCCI, F.; IULINI, B.; ACUTIS, P.; WANG, L.; LIANG, J.; WANG, M.; LI, X.; MONACO, S.; ZANUSSO, G.; ZOU, W.Q.; CARAMELLI, M.; GAMBETTI, P. Evalution of the human transmission risk of an atypical bovine spongiform encephalopathy prion strain. Journal of Virology, v.82, n.7, p.3697-3701, 2008.

KONOLD, T.; BONE, G.E.; CLIFFORD, D.; CHAPLIN, M.J.; CAWTHRAW, S.; STACK, M.J.; SIMMONS, M.M. Experimental H-type and L-type bovine spongiform encephalopathy in cattle: observation of two clinical syndromes and diagnostic challenges. Veterinary Research, v.8, p.22-33, 2012.

KONOLD, T.; PHELAN, L.J.; CLIFFORD, D.; CHAPLIN, M.J.; CAWTHRAW, S.; STACK, M.J.; SIMMONS, M.M. The pathological and molecular but not clinical phenotypes are maintained after second passage of experimental atypical bovine spongiform encephalopathy in cattle. BMC Veterinary Research, v.10, e.243, 2014.

LOMBARDI, G.; CASALONE, C.; D’ANGELO, A.; GELMETTI, D.; TORCOLI, G.; BARBIERI, I.; CORONA, C.; FASOLI, E.; FARINAZZO, A.; FIORINI, M.; GELATI, M.; IULINI, B.; TAGLIAVINI, F.; FERRARI, S.; CARAMELLI, M.; MONACO, S.; CAPUCCI, L.; ZANUSSO, G. Intraspecies transmission of BASE induces clinical dullness and amyotrophic changes. PLoS Pathogens, v.4, e 1000075, 2008.

MANUELIDIS, L. Transmissible encephalopathy agents: virulence, geography and clockwork. Virulence, v.1, p.101-104, 2010.

MASUJIN, K.; OKADA, H.; MIYAZAWA, K.; MATSUURA, Y.; IMAMURA, M.; IWAMARU, Y.; MURAYAMA, Y.; YOKOYAMA, T. Emergence of a novel bovine spongiform encephalopathy (BSE) prion from an atypical H-type BSE. Nature Scientific Report, v.6, 22753, 2016. DOI: $10.1038 /$ srep22753

MASUJIN, K.; SHU, Y.; YAMAKAWA, Y.; HAGIWARA, K.; SATA, T.; MATSUURA, Y.; IWAMARU, Y.; IMAMURA, M.; OKADA, H.; MOHRI, S.; YOKOYAMA, T. Biological and biochemical characterization of L-type-like bovine spongiform encephalopathy (BSE) in Japanese black cattle. Prion, v.2, p.123-128, 2008.

MELONI, D.; DAVIDSE, A.; LANGEVELD, J.P.M.; VARELLO, K.; CASALONE, C.; CORONA, C.; BALKEMA-BUSCHMANN, A.; GROSCHUP, M.H.; INGRAVALLE, F.; BOZZETTA, E. EU-approved rapid tests for bovine spongiform encephalopathy detect atypical forms: a study for their sensitivities. PLOSONE, v.7, e43133, 2012.

OKADA, H.; IWAMARU, Y.; IMAMURA, M.; MASUJIN, K.; MATSUURA, Y.; SHIMIZU, Y.; AKSAI, K.; MOHRI, S.; YOKOYAMA, T.; CZUB, $\mathrm{S}$. Experimental $\mathrm{H}$-type bovine spongiform encephalopathy characterized by plaques and glial- and stellate-type prion protein deposits. Veterinary Research, v.42, p.79-89, 2011.

ONO, F.; TASE, N.; KUROSAWA, A.; HIYAOKA, A.; OHYAMA, A.; TEZUKA, Y.; WADA, N.; SATO, Y.; TOBIUME, M.; HAGIWARA, K.;
YAMAKAWA, Y.; TERAO, K.; SATA, T. Atypical L-type bovine spongiform encephalopathy (L-BSE) transmission to Cynomolgus Macaques, a non-human primate. Japanese Journal of Infectious diseases, v.64, p.81-84, 2011.

ORGANIZAÇÃO MUNDIAL DE SAÚDE ANIMAL (OIE). BSE situation in the world and annual incidence rate. Paris: OIE, 2015. Disponível em: <http://www.oie.int/wahis_2/public/ wahid.php/Diseaseinformation/Immsummary >. Acesso em: 12 out. 2016.

ORGE, L.; MACHADO, C.G.; RAMALHO, L.; CARVALHO, R.; SILVA, J.; ALMEIDA, P.; TAVARES, P.; OCHOA, C.; LIMA, C.; PINTO, M.J.M.; SIMAS, J.P. Identification of H-type BSE in Portugal. Prion, v.9, p.22-28, 2015.

POLAK, M.P.; ROZEK, W.; ROLA, J.; ZMUDZINSKI, J.F. Prion protein glycoforms from BSE cases in Poland. Bulletin of Veterinary Institute of Pulawy, v.48, p.201-2015, 2004.

PRUSINER, S.B.; GADJUSEK, D.C.; ALPERS, M.P. Kuru with incubation periods exceeding two decades. Annals of Neurology, v.12, p.1-9, 1982.

RICHT, J.A.; KUNKLE, R.A.; ALT, D.; NICHOLSON, E.M.; HAMIR, A.N.; CZUB, S.; KLUGE, J.; DAVIS, A.J.; HALL, M. Identification and characterization of two bovine spongiform encephalopathy cases diagnosed in the United States. Journal of Veterinary Diagnostic Investigation, v.19, p.142-154, 2007.

SAEGERMAN, C.; SPEYBROECK, N.; ROELS, S.; VANOPDENBOSCH, E.; THIRY, E.; BERKVENS, D. Decision support tools for clinical diagnosis of disease in cows with suspected bovine spongiform encephalopathy. Journal of Clinical Microbiology, v.42, p. 172-178, 2004.

SALA, C.; MORIGNAT, E.; OUSSAIID, N.; GAY, E.; ABRIAL, D.; DUCROT, C.; CALAVAS, D. Individual factors associated with Land $\mathrm{H}$-type Bovine Spongiform Encephalopathy in France. BMC Veterinary Research, v.8, p.74-79, 2012.

SEUBERLICH, T.; BOTTERON, C.; WENKER, C.; CAFÉ-MARÇAL, V.; OEVERMANN, A.; HAASE, B.; LEEB, T.; HEIM, D.; ZURBRIGGEN, A. Spongiform encephalopathy in a miniature zebu. Emerging Infectious Diseases, v.12, n.12, p.1950-1953, 2006.

SEUBERLICH, T.; HEIM, D.; ZURBRIGGEN, A. Atypical transmissible spongiform encephalopathies in ruminants: a challenge for disease surveillance and control. Journal of Veterinary Diagnostic Investigation, v.22, p.823-842, 2010.

STACK, M. Western immunoblotting techniques for the study of transmissible spongiform encephalopathies. In: STACK, M. Methods and tools in biosciences and medicine - techniques in prion research. Berlim: Birkhäuser Verlag, 2004. p.97-116.

STACK, M.J.; FOCOSI-SNYMAN, R.; CAWTHRAW, S.; DAVIS, L.; CHAPLIN, M.J. Third atypical BSE case in Great Britain with H-type molecular profile. Veterinary Record, v. 165, p.605-606, 2009.

TERRY, L.A.; JENKINS, R.; THORNE, L.; EVEREST, S.J.; CHAPLIN, M.J.; DAVIS, L.A.; STACK, M.J. First case of H-type bovine spongiform encephalopathy in Great Britain. Veterinary Record, v.160, p.873-874, 2007. 
TORRES, J.M.; ANDREOLETTI, O.; LACROUX, C.; PRIETO, I.; LORENZO, P.; LARSKA, M.; BARON, T.; ESPINOSA, J.C. Classical bovine spongiform encephalopathy by transmission of $\mathrm{H}$-type prion in homologous prion protein context. Emerging Infectious Diseases, v.17, n.9, p.1636-1644, 2011.

WELLS, G.A.H.; SCOTT, A.C.; JOHNSON, C.T.; GUNNING, R.F.; HANCOCK, R.D.; JEFFREY, M.; DAWSON, M.; BRADLEY, R. A novel progressive spongiform encephalopathy in cattle. Veterinary Record, v.121, p.419-420, 1987.

WELLS, G.A.H.; WILESMITH, J.W. The neuropathology and epidemiology of bovine spongiform encephalopathy. Brain Pathology, v.5, p.91-103, 1995.

WILESMITH, J.W.; HOIVILLE, L.J.; RYAN, J.B.; SAYERS, A.R. Bovine spongiform encephalopathy: aspects of the clinical picture and analyses of possible changes 1986-1990. Veterinary Record, v.130, p.197-201, 1992.

WILESMITH, J.W.; WELLS, G.A.H.; CRANWELL, M.P.; RYAN, J.B. Bovine spongiform encephalopathy: epidemiological studies. Veterinary Record, v.123, p.638-644, 1988.

WILL, R.G.; IRONSIDE, J.W.; ZEIDLER, M.; ESTIBEIRO, K.; COUSENS, S.N.; SMITH, P.G., ALPEROVITCH, A.; POSER, S.; POCCHIARI, M.; HOFMAN, A. A new variant of Creutzfeldt-Jakob disease in UK. Lancet, v.347, p.921-925, 1996.

YAMAKAWA, Y.; HAGIWARA, K.; NOHTOMI, K.; NAKAMURA, Y.; NISHIJIMA, M.; HIGUCHI, Y.; SATO, Y.; SATA, Y. Atypical proteinase K-resistant prion protein ( $\operatorname{PrP}^{R E S}$ ) observed in an apparently healthy 23-month-old Holstein Steer. Japanese Journal of Infectious Diseases, v.56, p.221-222, 2003. 